\title{
Silicon-based III-V quantum dot devices for silicon photonics
}

\author{
Mingchu Tang, Siming Chen, Jiang Wu, Mengya Liao, Huiyun Liu* \\ Department of Electronic and Electrical Engineering, University College London, London, \\ WC1E 7JE, United Kingdom, \\ *huiyun.liu@ucl.ac.uk
}

Monolithically integrating III-V lasers on Si is the most promising solution to overcome the issue of lack of efficient light sources on Si platform. We demonstrated the first practical silicon-based telecommunications-wavelength InAs/GaAs quantum dot lasers with low threshold current density, high output power, high operation temperature and long lifetime. As one of the most wellestablished and high yield material, Si could offer high electronic and optical property, which has attracted researchers' attentions on the potential of opto-electronic integration circuits (OEIC). The advantages of using photon transmission rather than copper connection are large bandwidth, low crosstalk, and low power consumption. Although Si based modulator and detector have huge progress on development, but a lack of efficient and reliable optical amplifiers and laser sources on Si platform have been considered as "holy grail" due to the in-direct bandgap property of Si bulk materials [1]. In contrast, compound III-V semiconductor devices offer high efficiency as optical light emitting source and optical amplifier [2]. Integrating III-V materials on Si platform could combine the advantage of III-V materials and Si together, and hence create high-volume and lowcost OEIC.

The major issue of monolithic integration of IIIV on group IV platform is the formation of high density threading dislocations (TDs). The TDs are caused by the lattice mismatch between the III$\mathrm{V}$ materials and group IV substrates, for instance, GaAs has $4 \%$ lattice mismatch with Si. The propagation of TDs will cause high ratio of non-radiative recombination centre in III-V epitaxial active region. To stop the TD propagation, defects filter layers (DFLs) formed by InGaAs/GaAs strained-layer superlattices (SLSs) have been applied, which significantly reduce the density of TDs from $\sim 10^{10} / \mathrm{cm}^{2}$ at the interface between III-V and Si to $<10^{6} / \mathrm{cm}^{2}$ in III-V active region, as shown in Figure $1[3,4]$. As a zero-dimensional material, quantum dot (QD) has three-dimensional quantum confinements, which create delta-function like density of states. Therefore, QD lasers have low threshold currents, temperature insensitive operation, and less sensitivity to threading dislocations, which are the ideal candidate to form active region in III-V lasers grown on group IV substrates. High performance QD lasers grown on GaAs substrates have been well established, which proves its potential of growing on group IV platforms [2, 5-11].

In this work, we developed InAs/GaAs QD lasers monolithically grown on a Si substrate. The epitaxial materials were fabricated by a solid source molecular beam epitaxy system. Phosphorusdoped Si (100) wafer with $4^{\circ}$ offcut to the [011] plane was used. Prior to material growth, oxide desorption of silicon substrates was performed at $900{ }^{\circ} \mathrm{C}$ for 30 minutes. Epitaxy was then performed in the following order: a $6 \mathrm{~nm}$ AlAs nucleation layer, a $1 \mu \mathrm{m}$ GaAs buffer layer, InGaAs/GaAs dislocation filter layers, five layers of InAs/GaAs dot-in-well [DWELL] structures separated by $50 \mathrm{~nm}$ GaAs spacers in the middle of a $140 \mathrm{~nm}$ undoped GaAs waveguide between 
$1.4 \mu \mathrm{m}$ n-type lower and p-type upper $\mathrm{Al}_{0.4} \mathrm{Ga}_{0.6} \mathrm{As}$ cladding layers. Each DWELL structure consisted of a layer of 3- monolayer InAs QDs sandwiched by $2 \mathrm{~nm} \mathrm{In}{ }_{0.15} \mathrm{Ga}_{0.85} \mathrm{As}$ and $6 \mathrm{~nm}$ $\mathrm{In}_{0.15} \mathrm{Ga}_{0.85} \mathrm{As}$. The DWELLs were grown at $510{ }^{\circ} \mathrm{C}$ and $\mathrm{GaAs}$ and $\mathrm{AlGaAs}$ layers at $590{ }^{\circ} \mathrm{C}$. Finally, a $300 \mathrm{~nm}$ p-type GaAs contact layer was grown [5-11]. The broad-area lasers with $50 \mu \mathrm{m}$ wide stripes were fabricated by standard lithography and wet chemical etching techniques, the ridge was etched to roughly $100 \mathrm{~nm}$ above the active region, to give improved carrier confinement. Ti/Pt/Au and $\mathrm{Ni} / \mathrm{GeAu} / \mathrm{Ni} / \mathrm{Au}$ were deposited on the $\mathrm{p}+-\mathrm{GaAs}$ contact layer and exposed $\mathrm{n}+-\mathrm{GaAs}$ buffer layer to form the p-and n- contacts, respectively. After lapping the silicon substrate to 120 microns, the lasers were cleaved to the desired cavity lengths and mounted (as-cleaved) onto the heat-sink.

Low threshold current density $\left(\mathrm{J}_{\mathrm{th}}\right)$ and high output power have been demonstrated as shown in Figure 2 under $\mathrm{cw}$ operation at RT. A clear knee behaviour in the LI curve is observed at the lasing Jth of $62.5 \mathrm{~A} / \mathrm{cm}^{2}$, which corresponds to $12.5 \mathrm{~A} / \mathrm{cm}^{2}$ for each of the five QD layers. The lasing wavelength is at $1315 \mathrm{~nm}$, as shown in the inset of Figure 2. The output power measured from both facets is as high as $105 \mathrm{~mW}$ at an injection current density of $650 \mathrm{~A} / \mathrm{cm}^{2}$, with no evidence of power saturation up to this current density [3]. The ageing test was performed at a fixed temperature of $26{ }^{\circ} \mathrm{C}$, with the output power monitored for a constant $\mathrm{cw}$ drive current of $210 \mathrm{~mA}$. The ageing results are shown in Figure 3. An extrapolated mean time to failure (MTTF) of over 100,158 hours was demonstrated here [3].

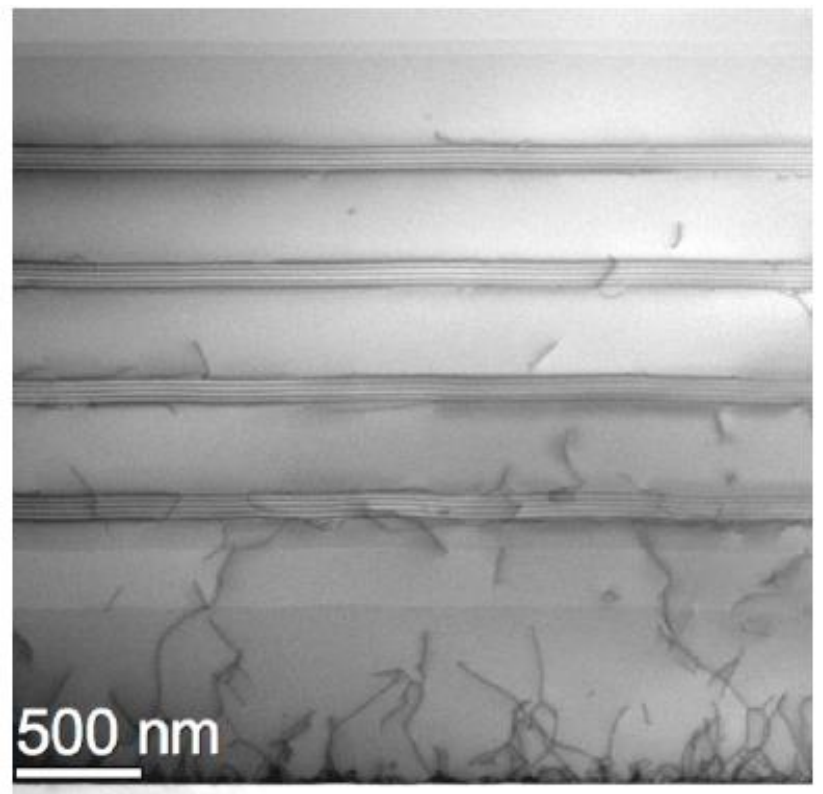

Figure 1: Bright field scanning scanning transmission electron microscopy image of GaAs buffer layer with InGaAs/GaAs dislocation filter layers. 


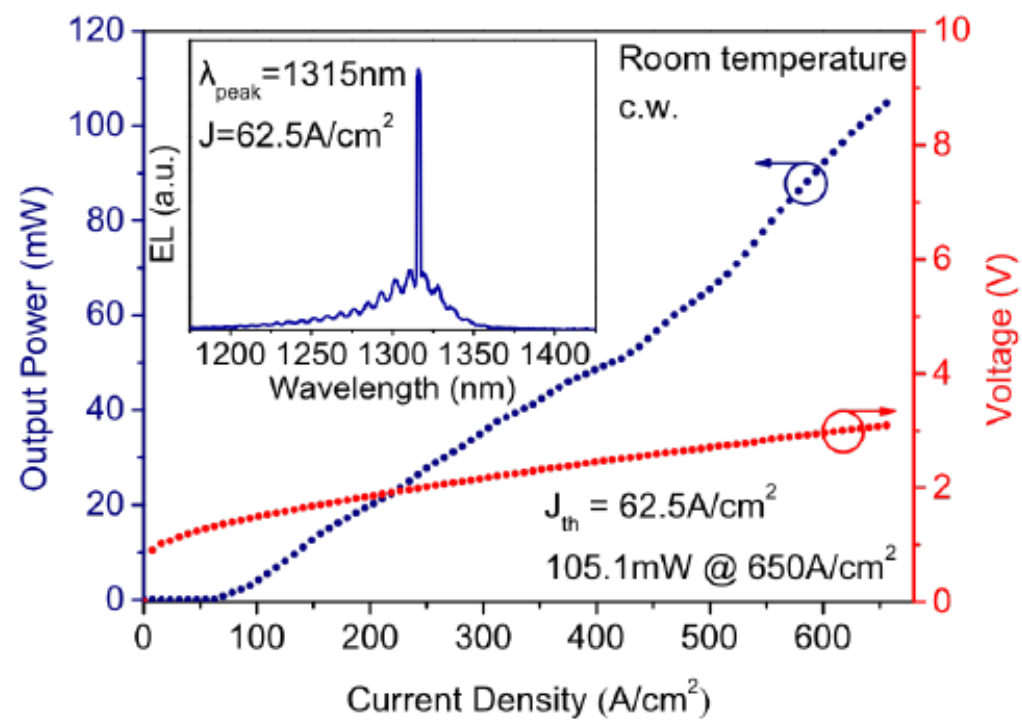

Figure 2: Light-current-voltage characteristics for a $50 \mu \mathrm{m} \times 3200 \mu \mathrm{m}$ InAs/GaAs QD laser grown on a silicon substrate under $\mathrm{cw}$ operation at $18{ }^{\circ} \mathrm{C}$.

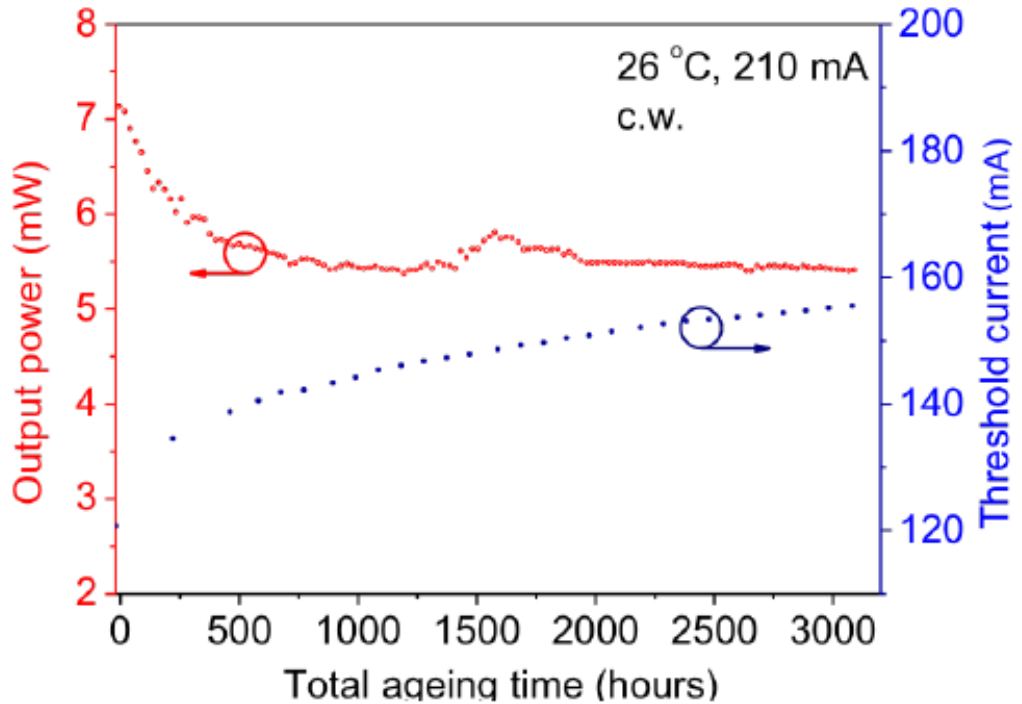

Figure 3: Ageing data for InAs/GaAs QD laser on Si at constant heat sink temperature of $26{ }^{\circ} \mathrm{C}$ and cw drive current of $210 \mathrm{~mA}$.

\section{Acknowledgements}

The authors acknowledge financial support from UK EPSRC under Grant No. EP/J012904/1.

\section{References}


[1] Liu, H., Wang, T., Jiang, Q., Hogg, R., Tutu, F., Pozzi, F. and Seeds, A., Nat. Photonics 5, 416419 (2011).

[2] Liu, H., Childs, D., Badcock, T., Groom, K., Sellers, I., Hopkinson, M., Hogg, R., Robbins, D., Mowbray, D., and Skolnick, M., IEEE Photon. Technol. Lett. 17, 1139-1141 (2005).

[3] Chen, S., Li, W., Wu, J., Jiang, Q., Tang, M., Shutts, S., Elliott, S., Sobiesierski, A., Seeds, J., Ross, I., Smowton, P., and Liu, H., Nat. Photonics [DOI: 10.1038/NPHOTON.2016.21].

[4] Tang, M., Chen, S., Wu, J., Jiang, Q., Dorogan, V.G., Benamara, M., Mazur, Y.I., Salamo, G.J., Seeds, A. and Liu, H., Opt. Express 22, 11528- 11535 (2014).

[5] Wang, T., Liu, H., Lee, A., Pozzi, F., and Seeds, A., Opt. Express 19(12), 11381-11386 (2011).

[6] Lee, A., Jiang, Q., Tang, M., Seed, A. and Liu, H., Opt. Express 20, 22181-22187 (2012).

[7] Lee, A.D., Jiang, Q., Tang, M., Zhang, Y., Seeds, A.J. and Liu, H., IEEE J. Sel. Top. Quantum Electron. 19, 1901107 (2013).

[8] Chen, S., Tang, M., Jiang, Q., Wu, J., Dorogan, V.G., Benamara, M., Mazur, Y.I., Salamo, G.J., Seeds, A. and Liu, H., Electron. Lett. 50, 1467- 1468 (2014).

[9] Tang, M., Wu, J., Chen, S., Jiang Q., Seeds, A., Liu, H., Dorogan, V.G., Benamara, M., Mazur, Y.I., Salamo, G., J. IET Optoelectronics, 61-64 (2015).

[10] Chen, S., Tang, M., Wu, J., Jiang, Q., Dorogan, V.G., Benamara, M., Mazur, Y.I., Salamo, G.J., Smowton, P., Seeds, A. and Liu, H., ACS Photonics 1, 638-642 (2014). 